\title{
Oscillatory connectivity as a diagnostic marker of dementia due to Alzheimer's disease
}

Musaeus, Christian Sandøe; Engedal, Knut; Høgh, Peter; Jelic, Vesna; Mørup, Morten; Naik, Mala; Oeksengaard, Anne Rita; Snaedal, Jon; Wahlund, Lars Olof; Waldemar, Gunhild

Total number of authors:

11

Published in:

Clinical Neurophysiology

Link to article, DOI:

10.1016/j.clinph.2019.07.016

Publication date:

2019

Document Version

Early version, also known as pre-print

Link back to DTU Orbit

Citation (APA):

Musaeus, C. S., Engedal, K., Høgh, P., Jelic, V., Mørup, M., Naik, M., Oeksengaard, A. R., Snaedal, J., Wahlund, L. O., Waldemar, G., \& Andersen, B. B. (2019). Oscillatory connectivity as a diagnostic marker of dementia due to Alzheimer's disease. Clinical Neurophysiology, 130(10), 1889-1899.

https://doi.org/10.1016/j.clinph.2019.07.016

\section{General rights}

Copyright and moral rights for the publications made accessible in the public portal are retained by the authors and/or other copyright owners and it is a condition of accessing publications that users recognise and abide by the legal requirements associated with these rights.

- Users may download and print one copy of any publication from the public portal for the purpose of private study or research.

- You may not further distribute the material or use it for any profit-making activity or commercial gain

- You may freely distribute the URL identifying the publication in the public portal 


\section{Journal Pre-Proof}

Oscillatory connectivity as a diagnostic marker of dementia due to Alzheim-

er's disease

Christian Sandøe Musaeus, Knut Engedal, Peter Høgh, Vesna Jelic, Morten

Mørup, Mala Naik, Anne-Rita Oeksengaard, Jon Snaedal, Lars-Olof Wahlund,

Gunhild Waldemar, Birgitte Bo Andersen

PII:

S1388-2457(19)31162-9

DOI:

https://doi.org/10.1016/j.clinph.2019.07.016

Reference:

CLINPH 2008927

To appear in: $\quad$ Clinical Neurophysiology

Received Date: $\quad 4$ January 2019

Revised Date: $\quad 26$ May 2019

Accepted Date: $\quad 3$ July 2019

Please cite this article as: Sandøe Musaeus, C., Engedal, K., Høgh, P., Jelic, V., Mørup, M., Naik, M., Oeksengaard, A-R., Snaedal, J., Wahlund, L-O., Waldemar, G., Bo Andersen, B., Oscillatory connectivity as a diagnostic marker of dementia due to Alzheimer's disease, Clinical Neurophysiology (2019), doi: https://doi.org/10.1016/j.clinph. 2019.07.016

This is a PDF file of an article that has undergone enhancements after acceptance, such as the addition of a cover page and metadata, and formatting for readability, but it is not yet the definitive version of record. This version will undergo additional copyediting, typesetting and review before it is published in its final form, but we are providing this version to give early visibility of the article. Please note that, during the production process, errors may be discovered which could affect the content, and all legal disclaimers that apply to the journal pertain.

(C) 2019 International Federation of Clinical Neurophysiology. Published by Elsevier B.V. All rights reserved. 


\section{Oscillatory connectivity as a diagnostic marker of dementia due to Alzheimer's disease}

Christian Sandøe Musaeus ${ }^{\mathrm{a}^{*}}$, Knut Engedal $^{\mathrm{b}}$, Peter Høgh ${ }^{\mathrm{c}}$, Vesna Jelic ${ }^{\mathrm{d}, \mathrm{e}}$, Morten Mørup $^{\mathrm{f}}$, Mala Naik ${ }^{\mathrm{g}}$, Anne-Rita Oeksengaard ${ }^{\mathrm{d}}$, Jon Snaedal ${ }^{\mathrm{h}}$, Lars-Olof Wahlund ${ }^{\mathrm{d}}$, Gunhild Waldemar ${ }^{\mathrm{a}}$, Birgitte Bo Andersen $^{\mathrm{a}}$

a Department of Neurology, Danish Dementia Research Centre (DDRC), Rigshospitalet, University of Copenhagen, Copenhagen, Denmark

${ }^{b}$ Norwegian National Advisory Unit on Ageing and Health (Ageing and Health), Vestfold Hospital Trust and Oslo University Hospital, Ullevaal, Oslo, Norway

${ }^{c}$ Regional Dementia Research Center, Department of Neurology, Zealand University Hospital, Roskilde, Denmark and Department of Clinical Medicine, University of Copenhagen, Copenhagen, Denmark

${ }^{d}$ Division of Clinical Geriatrics, Department of Neurobiology, Care Sciences and Society, Karolinska Institutet, Stockholm, Sweden

${ }^{e}$ Department of Geriatric Medicine, Memory Clinic, Karolinska University Hospital, Huddinge, Sweden

${ }^{f}$ Section for Cognitive Systems, DTU Compute, Technical University of Denmark, Lyngby, Denmark

${ }^{g}$ Department of Geriatric Medicine, Haraldsplass Deaconess Hospital, Bergen, Norway

${ }^{h}$ Department of Geriatric Medicine, Landspitali University Hospital, Reykjavik, Iceland

\section{* Corresponding author:}

Christian Sandøe Musaeus

Rigshospitalet, University of Copenhagen

Blegdamsvej 9 - Section 6911

2100 Copenhagen

Denmark

E-mail address: christian.sandoee.musaeus@,regionh.dk 


\begin{abstract}
Objective: Quantitative EEG power has not been as effective in discriminating between healthy aging and Alzheimer's disease as conventional biomarkers. But EEG coherence has shown promising results in small samples. The overall aim was to evaluate if EEG connectivity markers can discriminate between Alzheimer's disease, mild cognitive impairment, and healthy aging and to explore the early underlying changes in coherence.
\end{abstract}

Methods: EEGs were included in the analysis from 135 healthy controls, 117 patients with mild cognitive impairment, and 117 patients with Alzheimer's disease from six Nordic memory clinics. Principal component analysis was performed before multinomial regression.

Results: We found classification accuracies of above $95 \%$ based on coherence, imaginary part of coherence, and the weighted phase-lag index. The most prominent changes in coherence were decreased alpha coherence in Alzheimer's disease, which was correlated to the scores of the 10-word test in the Consortium to Establish a Registry for Alzheimer's Disease battery.

Conclusions: The diagnostic accuracies for EEG connectivity measures are higher than findings from studies investigating EEG power and conventional Alzheimer's disease biomarkers. Furthermore, decreased alpha coherence is one of the earliest changes in Alzheimer's disease and associated with memory function.

Significance: EEG connectivity measures may be useful supplementary diagnostic classifiers.

\title{
Keywords:
}

Coherence; Imaginary part of coherence; weighted phase-lag index; EEG; Diagnostic; Alzheimer's disease; Mild cognitive impairment. 


\section{Highlights}

- EEG connectivity showed diagnostic accuracies $>95 \%$ between patients and healthy controls.

- Decreased alpha coherence is one of the earliest changes in Alzheimer's disease.

- Alpha coherence is strongly associated with memory function.

\section{Abbreviations}

CERAD, Consortium to Establish a Registry for Alzheimer's Disease; MMSE, Mini-Mental State Examination 


\section{Introduction}

The diagnostic criteria for the dementia disorders of different etiologies are based on findings from clinical assessments but most of these criteria are not very helpful in the differential diagnosis in the very early phases of dementia (Reiman, 2014). The use of more advanced techniques like MRI, PET and cerebrospinal fluid biomarkers has proven very helpful in diagnosing Alzheimer's disease (Korf et al., 2004, Mosconi et al., 2009, Visser et al., 2009, Dubois et al., 2010, Resnick et al., 2010), but these methods are expensive, only performed in specialized hospitals, or invasive. EEG is widely available, and functional connectivity measures may serve as features for a diagnostic classifier.

Coherence, which is a method for calculating functional connectivity, refers to the temporal correlation between signals recorded from different brain regions. Only a few studies have shown that coherence alone may serve as a diagnostic marker in Alzheimer's disease, but promising results with sensitivity/specificity of 87\%/77\% (Adler et al., 2003) and 77.8\%/100\% (Jelic et al., 1996) have been demonstrated. These two studies, however, had a small sample size and used single coherence values for classification (Jelic et al., 1996, Adler et al., 2003). A larger study partly used coherence values and found an area under the curve of the receiver operating characteristic curve of 0.90 when separating Alzheimer's disease from healthy controls (Engedal et al., 2015). However, no studies have used coherence to separate both patients with mild cognitive impairment and Alzheimer's disease from healthy controls. In addition, recent evidence has suggested that volume conduction through the tissue can obscure the results when applying coherence. To overcome this problem, studies have found that the imaginary part of coherence is insensitive to volume conduction (Grosse et al., 2002, Nolte et al., 2004). However, the imaginary part of coherence is still affected by the signal amplitudes, which may make it more sensitive to noise (Vinck et al., 2011). Recent studies have therefore suggested that connectivity measurements solely based on the phase relationship may be less sensitive to volume conduction as measured with the weighted phase-lag index (Vinck et al., 
2011, Hardmeier et al., 2014). So far, no studies have to our knowledge tested if coherence performs as well as the imaginary part of coherence or the weighted phase-lag index as features for classification.

Multiple studies have investigated the changes in coherence in patients with Alzheimer's disease compared to healthy controls, with the most common finding being decreased alpha coherence (Leuchter et al., 1987, Leuchter et al., 1992, Cook and Leuchter, 1996, Locatelli et al., 1998, Wada et al., 1998, Jelic et al., 2000, Knott et al., 2000, Adler et al., 2003, Jeong, 2004, Sankari et al., 2011, Wang et al., 2014). Notwithstanding, the mentioned studies may have recruited patients with Alzheimer's disease at a later stage of the disease, as indicated by the low mean for the Mini-Mental State Examination (MMSE) of 15.6-21.9, with one study reporting an MMSE range of 11.7-14.0 (Wang et al., 2014). In addition, only one study included patients with mild cognitive impairment (Jelic et al., 2000). To understand the underlying mechanism behind coherence as a diagnostic marker, early coherence changes need to be further explored.

Only few studies have investigated the association between coherence and cognitive scores (Leuchter et al., 1992, Dunkin et al., 1994, Knott et al., 2000, Chen et al., 2015), and no studies have looked at associations with cerebrospinal fluid biomarkers. This may largely be due to the multiple comparisons problem, if done for each connection. Another possible approach to overcoming this issue is to correlate the cognitive tests or cerebrospinal fluid biomarkers to total coherence (Babiloni et al., 2010), which is the sum of all connections for each frequency band.

As a result, the current exploratory study tests whether coherence, imaginary part of coherence or the weighted phase-lag index may be used as features for classification between Alzheimer's disease, mild cognitive impairment, and healthy aging. We used the same protocol for the diagnostic classifier as previously reported (Musaeus et al., 2018a). To understand the early changes in coherence in 
dementia due to Alzheimer's disease, we also wanted to examine the differences in coherence between patients with Alzheimer's disease, mild cognitive impairment, and healthy controls. Lastly, to assess the functional relevance of coherence, we tested whether total alpha coherence was correlated to either cognitive scores or biomarkers. 


\section{Methods and materials}

\subsection{Participants}

The data from the current study has been used in previous publications (Engedal et al., 2015) and some of the subjects have also participated in another study (Musaeus et al., 2018b). The results from the power analysis derived from the frequency spectrum are presented elsewhere (Musaeus et al., 2018a). The patients and the healthy controls were recruited from six Nordic memory clinics. The data was originally collected as part of a validation study (Engedal et al., 2015), which entailed ensuring that each diagnostic group comprised about 20 patients, a common requirement in validation studies. A minimum of 60 patients and 20 healthy controls were recruited at each site in the validation study. All 365 of the included study participants were consecutively recruited in most clinics during their first assessment using the following predefined exclusion criteria: 1) neurological disorders with dementia other than Alzheimer's disease, Parkinson's disease dementia and Lewy body dementia; 2) major psychiatric disorders; and 3) alcohol or drug abuse. The 146 healthy controls were recruited from among patients' family members, employees at the recruiting hospitals, or through advertising.

Due to the low number of patients with Lewy body dementia $(n=10)$, vascular dementia $(n=15)$, Parkinson's disease dementia $(n=5)$, frontotemporal dementia $(n=4)$, mixed Alzheimer's disease and vascular dementia $(n=5)$, and mixed dementias $(n=8)$, they were excluded from the analysis. Patients with subjective cognitive decline $(n=64)$ were excluded in the analysis in this report. Due to either poor quality or lost EEGs, we had to exclude eight EEGs from healthy controls, eight from patients with mild cognitive impairment, and 15 from patients with Alzheimer's disease. Lastly, three healthy controls were excluded due to use of either antidepressants or antipsychotics since the underlying condition and/or severity was unknown (see flow diagram in Fig. 1). Table 1 presents a full description of the included patients and healthy controls. 


\subsection{Clinical diagnostic assessment}

All patients were examined according to each memory clinic's standards, which were similar. Clinical assessment comprised: 1) a history from the patient and an informant; 2) a physical examination focusing on neurological and cardiology status; 3) blood tests to screen for disorders that could be associated with cognitive impairment; 4) neuropsychological tests covering various cognitive domains; 5) CT or MRI of the brain to evaluate white matter changes, general atrophy, and atrophy of the medial temporal lobes; and 6) a lumbar puncture to examine amyloid beta-42 and total tau and phosphorylated tau protein in the cerebrospinal fluid, when indicated. Some patients were assessed with fludeoxyglucose PET or Tc-HMPAO SPECT. The neuropsychological tests for the initial examination differed slightly between the clinics but included both verbal and visuospatial memory assessments. All the patients included in this study underwent MMSE (Folstein et al., 1975), the Clock-Drawing Test (Shulman, 2000), and the 10-word tests of the Consortium to Establish a Registry for Alzheimer's disease (CERAD) (Morris et al., 1988). A previously published paper contains the details of the clinical assessments (Braekhus et al., 2011).

The clinical diagnoses were made at consensus meetings with all doctors present at each memory clinic, or by at least two experienced doctors, and applied the Diagnostic and Statistical Manual of Mental Disorders, Fourth Edition Text Revision and the McKhann criteria for the diagnosis of Alzheimer's disease (McKhann et al., 1984). Winblad criteria were used to diagnose mild cognitive impairment (Winblad et al., 2004). All diagnoses were made blinded to the EEG results. After undergoing a cognitive test battery to rule out cognitive impairment, the healthy controls were interviewed and histories of previous and present disorders and drug use were recorded. Any individuals who had a cognitive test result below one standard deviation, according to their age, were excluded. 


\subsection{EEG recording}

EEGs were recorded using NicoletOne EEG Systems $\left(\right.$ Natus $\left.^{\circledR}\right)$. The International 10-20 System of Electrode Placement was used for the 19 electrodes (Supplementary Figure S1), and the features were evaluated using the average montage. Two bipolar electro-oculography channels and one electrocardiogram channel were recorded to monitor artefacts. Most EEGs were recorded alternating between three-minute periods of eyes closed and eyes open. The participants were alerted if they became visibly drowsy.

Some of the clinics participating in the study did not record segments with eyes open. As a result, the number of segments with eyes closed and eyes open for each group was: healthy controls (eyes closed: 138, eyes open: 110); Alzheimer's disease (closed: 117, open: 97); and mild cognitive impairment (closed: 117, open: 99).

\subsection{Pre-processing EEG}

The data, imported to MATLAB (Mathworks, v2016a) using the EEGLAB toolbox (Delorme and Makeig, 2004), was divided into eyes-closed and eyes-open segments using the events in the EEG during recording. Some of the EEGs did not contain eyes-open segments, and the eyes-closed segments were selected from the first 10 minutes of the recording to prevent inclusion of segments with drowsiness or sleep. The electrodes were computationally located on the scalp by means of the DIPFIT toolbox (Oostenveld et al., 2011). The excessive channels (like electrocardiogram and reference electrodes) were removed and the data was bandpass-filtered from 1-70 $\mathrm{Hz}$ using the pop_firws function in MATLAB, with a filter order of two, and the Kaiser window parameter beta was estimated using a maximum passband ripple of 0.001 . Afterwards, the data was band-stop filtered from $45-55 \mathrm{~Hz}$ using the same settings. The data was subsequently down-sampled to $200 \mathrm{~Hz}$, if the 
sampling rate was $>200 \mathrm{~Hz}$. Next, the data was divided into one-second epochs and the EEGs were visually inspected to remove excessive noise or artefacts. Channels with excessive noise, drift, or a bad connection were interpolated using spherical interpolation. The EEG had to have $\leq 3$ electrodes with excessive artefacts; otherwise, the EEG was excluded from the analysis. Afterwards, independent component analysis was performed using the extended infomax algorithm (Lee et al., 1999), extracting up to 19 components based on the data rank. This was done for each file, and components containing eye blinks, eye movement, electrocardiogram artefacts, or specific line-noise artefacts were removed. Lastly, the EEGs were visually inspected again, and epochs with excessive noise or artefacts were removed. The investigator was blinded to diagnosis.

\subsection{Connectivity calculations}

Coherence between two signals was calculated as the square of the cross spectrum of the electrodes divided by the product of the power spectra of the individual electrodes. This way of calculating coherence is a measure of the consistency of a phase relationship between two signals ranging from 0 to 1 . Coherence was calculated between each pair of electrodes for each of the following frequency bands: delta (1-3.99 Hz); theta (4-7.99 Hz); alpha (8-12.99 Hz); and beta (13-29.99 Hz), together with beta1 (13-17.99 Hz); beta2 (18-23.99 Hz); and beta3 $(24-29.99 \mathrm{~Hz})$, for eyes closed and open. The coherence values were calculated for each epoch separately and, afterwards, we calculated the mean across epochs. This was performed with the assumption that the phase relationship would be constant across epochs. Total coherence was calculated by averaging across all connections and was done separately for each frequency band and for eyes closed and open.

The imaginary part of coherence was calculated by taking the imaginary part of the cross-spectrum of the electrodes divided by the square root of the product of the power spectra of the individual 
electrodes (Nolte et al., 2004). Since we were interested in the magnitude as calculated for coherence, we calculated the absolute value of the imaginary result. To calculate the weighted phase-lag index, we applied the same approach as previously described (Vinck et al., 2011). Here, the weighted phaselag index was calculated across trials. Both the imaginary part of coherence and the weighted phaselag index was calculated for the same frequency bands as described for coherence.

\subsection{Prediction}

Prediction analysis, as previously executed in the context of spectral power (Musaeus et al., 2018a), was performed in eyes closed and open for the three diagnoses (Alzheimer's disease, mild cognitive impairment, and healthy controls). The data set, which consisted of the number of subjects using 171 coherence values (19 electrodes) and seven frequency bins (delta, theta, alpha, beta1, beta2, beta3, and beta), was turned into a data matrix of number of subjects by $7 * 171$ coherence features and compressed using principal component analysis, such that $99 \%$ of the data variance was kept for the subsequent classification analysis. For the classification, we used multinomial regression for the three-class classification of Alzheimer's disease, mild cognitive impairment, and healthy controls, as implemented using the minFunc optimization procedure (Schmidt, 2005). We quantified the model performance using leave-one-out cross-validation. The classification accuracies are reported averaged over the number of observations (i.e., subjects) left out one at a time in the leave-one-out cross-validation procedure. The same procedures were performed for the imaginary part of coherence, and for the weighted phase-lag index. As a post-hoc analysis, we employed only alpha coherence as a diagnostic classifier using the same approach described above. 


\subsection{Statistics}

All statistics were performed in MATLAB (vR2016a). To compare sex, we performed a chi-square test. For age, years of education, the MMSE, the ten-word list of the Consortium to Establish a Registry for Alzheimer's Disease score and sub-scores (learning, recognition, and recall), and the number of 1-second epochs, we performed as a one-way ANOVA for three groups: Alzheimer's disease, mild cognitive impairment, and healthy controls. The comparison was significant with a pvalue $<0.05$.

When comparing the coherence values, we first performed Fisher's Z transformation before any statistical test due to the non-normally distributed data. For comparing Alzheimer's disease, mild cognitive impairment, and healthy controls, we performed an ANCOVA using age, sex, years of education, and current medication as covariates. Current medications were included as binary values for whether the person received antipsychotics, antidepressants, hypnotics, anti-dementia drugs, or painkillers. To correct for multiple comparisons, we performed Bonferroni-Holm correction for 171 connections and seven frequency bands, which is equal to 1197 comparisons times two for eyes closed and open. Afterwards, we computed post-hoc t-tests without covariates on the Z-transformed data if the group comparison was significant after Bonferroni-Holm correction (corrected p-value $<0.05)$. The post-hoc t-tests were considered significant with a p-value $<0.001$. The p-value was set at 0.001 for the post-hoc test to correct for multiple comparisons at the post-hoc level. For visualization of the connectivity, we used the BrainNet Viewer toolbox (Xia et al., 2013) with the significant t-values from the post-hoc analyses. The same approach was performed for both the imaginary part of coherence, and the weighted phase-lag index.

For correlation analyses, we performed a Spearman correlation between total alpha coherence when the participants had eyes closed and the total Consortium to Establish a Registry for Alzheimer's 
Disease (CERAD) score, including the sub-scores learning, recall, and recognition, and the Alzheimer's disease biomarkers (amyloid, total tau, and phosphorylated tau). 


\section{Results}

\subsection{Demographics}

Table 1 presents a description of the demographics and comparisons between groups for patients with Alzheimer's disease, mild cognitive impairment, and healthy controls. The number of 1-second epochs between Alzheimer's disease $(\operatorname{mean}(\mathrm{SD})=130.82(53.14))$, mild cognitive impairment $(\operatorname{mean}(\mathrm{SD})=119.64(48.23))$, and healthy controls $(\operatorname{mean}(\mathrm{SD})=113.93(55.49))$ was significantly different $(\mathrm{p}$-value $=0.038, \mathrm{~F}=3.3072$, degrees of freedom $=366)$ looking at all the included participants.

\subsection{Diagnostic accuracy}

We found a classification accuracy of $95.9 \%$ (specificity $=94.07 \%$, sensitivity for mild cognitive impairment $=97.44 \%$, sensitivity for Alzheimer's disease $=96.58 \%$ ) when comparing all three patient groups (Alzheimer's disease, mild cognitive impairment, and healthy controls) for all coherence values with eyes closed (Fig. 2A). When the participants had open eyes, the accuracy was $98.0 \%$ ( specificity $=97.20 \%$, sensitivity for mild cognitive impairment $=96.97 \%$, sensitivity for Alzheimer's disease $=100 \%$ ) for all three patient groups for all coherence values (see Fig. 2D).

For imaginary part of coherence for the eyes-closed segment, we found an accuracy of $96.7 \%$, ( specificity $=95.55 \%$, sensitivity for mild cognitive impairment $=96.58 \%$, sensitivity for Alzheimer's disease $=98.29 \%$ ), and $98.3 \%$ (specificity $=98.13 \%$, sensitivity for mild cognitive impairment $=$ 96.97\%, sensitivity for Alzheimer's disease $=100 \%$ ) in eyes-open condition (Figs. 2B and 2E).

For the weighted phase-lag index for the eyes-closed segment, we found an accuracy of $96.2 \%$, ( specificity $=97.78 \%$, sensitivity for mild cognitive impairment $=92.31 \%$, sensitivity for Alzheimer's 
disease $=98.29 \%)$, and $98.3 \%$ (specificity $=100 \%$, sensitivity for mild cognitive impairment $=$ 95.96\%, sensitivity for Alzheimer's disease $=98.97 \%$ ) in eyes-open condition (Figs. 2C and 2F).

As a post-hoc analysis, we also calculated the classification accuracy for alpha coherence between the three groups with eyes closed. We found an accuracy of $87.5 \%$ (specificity $=84.44 \%$, sensitivity for mild cognitive impairment $=88.03 \%$, sensitivity for Alzheimer's disease $=90.60 \%$; see Fig. $2 \mathrm{G}$.

Since the number of 1-second epochs was significantly different between the three groups, we examined whether there was any confounding effect (Supplementary Material, Possible confounders of the classifier, pp. 30-36). We found that the number of epochs was unable to predict the diagnoses. When similarly dividing the participants into three groups with an equal number of participants (below 94 epochs, between 94 to 131 epochs, and above 131 epochs), we found that the participants with over 131 epochs performed worse $($ accuracy $=81.3 \%)$ than the group with 94 to 131 epochs (95.1\%), and the group with less than 94 epochs (97.6\%). Furthermore, when only the first 94 epochs were included for the group with 94 to 131 epochs, we found a poor classification accuracy (42.6\%). We also observed that systematically reducing the number of included participants reduced classification performance. No difference in accuracy was found when the variance preserved by the principal component analysis was above $90 \%$.

\subsection{Coherence - eyes closed}

For coherence, the most prominent findings were decreases for all significant electrode pairs in the alpha band for Alzheimer's disease compared to healthy controls and mild cognitive impairment (Fig.

3) For mild cognitive impairment compared to healthy controls, the decreases were less pronounced. Between patients with Alzheimer's disease and healthy controls in the theta band, we found significant increases in coherence for almost all electrode pairs, except between $\mathrm{Cz}-\mathrm{Fz}, \mathrm{Cz}-\mathrm{T} 3, \mathrm{Cz}-$ 
T5, Cz-T4, and T6-O2. When comparing mild cognitive impairment and healthy controls in the theta band, we found increases in coherence for most electrode pairs, except for decreased left temporal, parietal, and occipital connections, corresponding to the right hemisphere. For all the beta bands, we found increases and decreases between Alzheimer's disease and healthy controls. However, patients with mild cognitive impairment showed a pattern of decreased coherence compared to both healthy controls and patients with Alzheimer's disease, which is also the case when examining total coherence (Fig. 4). For significant differences in coherence in eyes closed, see Fig. 3 and, for total coherence, see Fig 4. Supplementary Figures S2-3 contain colourmaps, while Supplementary Tables S1-S14 and Tables S29-S35 present statistical results. For significant differences for eyes closed for the imaginary part of coherence see Supplementary Figure S11 and for the weighted phase-lag index see Supplementary Figure S13.

\subsection{Coherence - eyes open}

The most prominent findings for eyes open were in the delta and theta bands (Figs. 5 and 6). In the delta band, we found an overall increase in coherence for patients with Alzheimer's disease and a decrease for patients with mild cognitive impairment compared to healthy controls. In the theta band, we found an increase in the majority of the coherence values for patients with Alzheimer's disease and patients with mild cognitive impairment compared to healthy controls. The most prominent connections were found between frontal electrodes and between frontal and temporal electrodes (T3F8, T4-F7 and F8-F3). In the beta1 and beta2 bands, we found that patients with mild cognitive impairment had a decreased coherence compared to patients with Alzheimer's disease and to healthy controls (Figs. 5 and 6). Supplementary Figures S4-S5 contain colourmaps, while Supplementary Tables S15-S28 and Tables S36-S42 present statistical results. For significant differences for eyes 
open for the imaginary part of coherence see Supplementary Figure S12 and for the weighted phaselag index see Supplementary Figure S14.

\subsection{Association with cognitive scores and Alzheimer's disease biomarkers}

We found that total alpha coherence was significantly correlated to the CERAD score (p-value $<0.000$, rho $=0.4438)$, including the sub-scores for learning $(\mathrm{p}$-value $<0.000$, rho $=0.4269)$, delayed recall $(\mathrm{p}$-value $<0.000$, rho $=0.4304)$, and recognition $(\mathrm{p}$-value $<0.000$, rho $=0.3109)$. In addition, we found that total alpha coherence was significantly correlated with the MMSE score (p-value $<0.000$, rho $=0.3991)$. No significant correlations were found between Alzheimer's disease biomarkers and total alpha coherence. Supplementary Figure S4 shows the scatterplots for the significant correlations. 


\section{Discussion}

In the present study, we found accuracies above 95\% when separating Alzheimer's disease, mild cognitive impairment, and healthy controls using either EEG coherence, the imaginary part of coherence, or the weighted phase-lag index. Furthermore, when only using the alpha band coherence, we find an accuracy of $87.5 \%$. The most prominent early coherence changes were decreased alpha in Alzheimer's disease and, to a lesser extent, for patients with mild cognitive impairment compared to healthy older controls in eyes closed condition. We found that total alpha coherence was positively correlated with the CERAD 10-word list scores. Furthermore, we found an overall decreased beta coherence in patients with mild cognitive impairment compared to both Alzheimer's disease and healthy controls.

Two previous studies examining EEG coherence to discriminate between Alzheimer's disease and healthy aging showed a sensitivity/specificity of $87 \% / 77 \%$ (Adler et al., 2003) and $77.8 \% / 100 \%$ (Jelic et al., 1996), but they had a low number of participants, used single coherence values for the classification, and did not include mild cognitive impairment. One study had 31 participants with Alzheimer's disease and 17 who were cognitively unimpaired and depressed (Adler et al., 2003), while the other had less than 20 participants in each group (Jelic et al., 1996). In the current study, we used a predefined protocol (Musaeus et al., 2018a) and performed principal component analysis, which included all frequency bands into one prediction analysis and the study was conducted in a large population. Compared to conventional EEG measurements, we previously found in the same population that EEG power was unable to separate Alzheimer's disease, mild cognitive impairment, and healthy controls (accuracies between 48.0\%-53.5\%) (Musaeus et al., 2018a). Furthermore, other studies using EEG power have found promising results (Claus et al., 1999, Lehmann et al., 2007, Snaedal et al., 2012) but had low sample size (Claus et al., 1999) or were not able to replicate the findings (Ommundsen et al., 2011). However, more advanced EEG techniques, such as feature 
selection algorithms (Trambaiolli et al., 2017), entropy (Abasolo et al., 2005), and amplitude modulation (Fraga et al., 2013), have shown promising results but with low sample sizes. In addition, the accuracies above 95\% are higher than conventional Alzheimer's disease biomarkers, including fludeoxyglucose PET, cerebrospinal fluid biomarkers, or MRI, which has been shown to be $>80 \%$ (Frisoni et al., 2013). Consequently, we suggest that quantitative EEG coherence can serve as a supplemental classifier to differentiate between Alzheimer's disease, mild cognitive impairment, and healthy controls. In further support of this, we found that using imaginary part of coherence, and the weighted phase-lag index, which both should be less affected by volume conduction, had accuracies close to coherence. When including only alpha coherence, which constituted the most pronounced changes, we found an accuracy of $85.1 \%$. This indicates that including other frequency bands may increase the diagnostic accuracy. These are very promising findings, but future studies are needed to validate them, and other groups of neurodegenerative diseases should possibly be added to the diagnostic classifier. Furthermore, to our knowledge, no studies have used coherence with eyes open to predict diagnosis of dementia due to Alzheimer's disease, which is useful since patients with closed eyes may get drowsy or fall asleep during the EEG recording. Furthermore, this is the first study using the imaginary part of coherence, and the weighted phase-lag index as a classifier of disease in patients with Alzheimer's disease.

We also studied any potential confounding effect of the number of trials included (see Supplementary Material, Possible confounders of the classifier, pp. 30-36). We found that the number of epochs was unable to predict the diagnoses. However, we found that the classifier performed worse in the group that had more than 131 epochs compared to the groups with lengths less than 131 epochs. This may indicate that either the patients with larger datasets, and therefore the ability to comply with the protocol, were in a milder stage of the disease or even misdiagnosed, or that we included sleep during the preprocessing. Furthermore, we were unable to reproduce the diagnostic accuracy of $95.1 \%$ in the 
group with 94 to 131 epochs when only the first 94 epochs were included, with an accuracy of only $42.6 \%$ achieved. This suggests that reducing the signal-to-noise ratio (by reducing the number of epochs) has a profound effect on the classification accuracy. We investigated the performance when reducing the amount of variance preserved to $70 \%, 80 \%, 90 \%, 95 \%, 97.5 \%$, and $99 \%$ and observed that classification performance declined when preserving less than $95 \%$ of the variance, but the performance saturated when $95 \%$ or more of the variance was preserved. This indicates that discriminatory signals in the low variance components do not have any considerable effect on classification accuracy. By systematically reducing the number of participants, we observed that the classification performance was reduced but, even when only including 100 subjects, we achieved a high classification performance of well above $80 \%$ when preserving $95 \%$ or more of the variance in the principal component analysis. Future studies should thus record larger EEG datasets and systematically test whether the effect of epochs on classification performance is driven by changes in the signal-to-noise ratio.

The most prominent coherence change in Alzheimer's disease was a large decrease in alpha coherence compared to both mild cognitive impairment and healthy controls with eyes closed at the time of diagnosis (Figs. 3 and 4), which is in line with previous research (Leuchter et al., 1987, Leuchter et al., 1992, Cook et al., 1996, Locatelli et al., 1998, Wada et al., 1998, Jelic et al., 2000, Knott et al., 2000, Adler et al., 2003, Jeong, 2004, Sankari et al., 2011, Wang et al., 2014) (Fig. 3). The underlying mechanism for decreased alpha coherence in Alzheimer's disease is still controversial but has been attributed to a decrease in cholinergic connectivity (Locatelli et al., 1998, Adler et al., 2003), which is explained by decreased alpha coherence after administration of anticholinergic drugs like scopolamine (Sloan et al., 1992, Bajo et al., 2015). In further support of this, decreased alpha band coherence has also been associated with the ApoE4 genetic risk factor (Jelic et al., 1997), and studies have found that cholinesterase inhibitors lead to greater cognitive improvement in patients 
with the ApoE4 genetic risk factor (Bizzarro et al., 2005, Petersen et al., 2005, Choi et al., 2008, Patterson et al., 2011), which may indicate a more pronounced decrease in cholinergic function. In patients with mild cognitive impairment, we also showed a decrease in alpha coherence, which is in line with previous studies (Jelic et al., 1996, Moretti et al., 2008, Bian et al., 2014), but it was not as pronounced as in Alzheimer's disease. We hypothesize that the underlying degenerative processes of the cholinergic system may have led to the decreased alpha coherence, which is not as pronounced in patients with mild cognitive impairment and supports the idea of coherence as a classifier of disease. However, studies investigating the EEG effect of cholinesterase inhibitors in patients with Alzheimer's disease are needed. Furthermore, we found positive significant correlations between cognitive scores and total alpha coherence, and especially the total CERAD score ( $p$-value $<0.000$, rho $=0.4438)$ and delayed recall $(p$-value $<0.000$, rho $=0.4304)$. A recent study showing that longitudinal changes in cognition were correlated to C3-C4 and P3-P4 alpha coherence support this finding (Chen et al., 2015). These results combined point to decreased alpha coherence as a hallmark of early Alzheimer's disease. Unfortunately, the PET data was not available for the current study.

Previous studies found very heterogeneous results in the eyes closed condition in the delta and theta bands, with decreases and increases in coherence (Leuchter et al., 1987, Locatelli et al., 1998, Adler et al., 2003, Brunovsky et al., 2003, Sankari et al., 2011, Wang et al., 2014), with most studies finding a general decrease in coherence. In the delta band, studies found no differences using global scores (Besthorn et al., 1994, Jelles et al., 2008), specific increases (Locatelli et al., 1998) or decreases (Sankari et al., 2011) between electrode pairs in patients with Alzheimer's disease compared with healthy controls, which can explain the mixed results in the current study (Fig. 3). In the theta band, some studies found a decrease (Besthorn et al., 1994, Sankari et al., 2011), but this may be due to a small sample size. We found an overall increase in theta coherence (Fig. 3), which can partially be explained as an overall increase in spectral theta power, as previously reported with the same dataset 
(Musaeus et al., 2018a). We also found decreased coherence in the beta bands in mild cognitive impairment compared to both Alzheimer's disease and healthy controls (Fig. 3, and Fig. 4), which has not previously been reported and needs to be explored further. We also investigated the eyes open condition, which, to our knowledge, has not been investigated previously, and found that the most pronounced changes were in the theta and delta bands (Fig 5). The increased theta band coherence in patients with Alzheimer's disease and patients with mild cognitive impairment compared to healthy controls, which we also found in the eyes closed condition, may be related to our previous finding of increase spectral theta power (Musaeus et al., 2018a). The current study has some limitations; for example, the lack of follow-up data in the mild cognitive impairment group prevented us from investigating mild cognitive impairment with Alzheimer's disease pathology. In addition, we decided not to include the gamma frequency band in the analysis since the EEGs in the current study were recorded in a clinical setting, which included high-frequency noise that especially affected the gamma band. Furthermore, the coherence measure may be subject to common feeding effects (Dauwels et al., 2010, Blinowska and Kaminski, 2013), possibly leading to spurious connections, which is why any conclusions regarding specific connections have been avoided. Demographically, we found that the healthy controls were younger than the Alzheimer's disease and mild cognitive impairment groups, and a proportion of Alzheimer's disease and mild cognitive impairment patients were being treated with medications that may have affected the EEG. Furthermore, the healthy control group had a higher level of education than either clinical group, which may also have played a role in memory impairment. However, we tried to correct for these confounders by including age, medication, and education as covariates when performing ANCOVA. The current study, which is the largest prospective multi-center study to date with consecutively recruited patients at an early stage of Alzheimer's disease applying coherence, imaginary part of coherence, and weighted phase-lag index, 
found accuracies $>95 \%$ when predicting Alzheimer's disease, mild cognitive impairmentand healthy aging, which is very promising.

\section{Conclusions}

The current study found that coherence, the imaginary part of coherence, and the weighted phase-lag index are very promising classifiers (accuracies $>95 \%$ ) for Alzheimer's disease, mild cognitive impairment, and healthy aging, because the accuracies are higher than for both quantitative EEG power and conventional biomarkers like MRI, fludeoxyglucose PET, and cerebrospinal fluid biomarkers. Furthermore, in patients with Alzheimer's disease, we found the largest decrease in alpha coherence and, to a lesser extent, for patients with mild cognitive impairment at the time of diagnosis, which was positively correlated with memory function. We hypothesize that the decreased alpha coherence is the EEG coherence hallmark of early Alzheimer's disease. These findings suggest that EEG coherence, imaginary part of coherence, and the weighted phase-lag index may be used as supplementary diagnostic tools. However, a validation of the classifier in a separate cohort is needed.

\section{Conflict of interest}

None.

\section{Acknowledgments}

We would like to thank the Kavli Charitable Trust in Bergen, Norway, which funded this EEG study. We would also like to thank all the doctors, nurses, and research assistants of the NIDD working group for their valuable contributions to the NORD EEG study. 


\section{References}

Abasolo D, Hornero R, Espino P, Poza J, Sanchez Cl, de la Rosa R. Analysis of regularity in the EEG background activity of Alzheimer's disease patients with Approximate Entropy. Clin Neurophysiol. 2005;116:1826-34.

Adler G, Brassen S, Jajcevic A. EEG coherence in Alzheimer's dementia. J Neural Transm (Vienna). 2003;110:1051-8.

Babiloni C, Frisoni GB, Vecchio F, Pievani M, Geroldi C, De Carli C, et al. Global functional coupling of resting EEG rhythms is related to white-matter lesions along the cholinergic tracts in subjects with amnesic mild cognitive impairment. J Alzheimers Dis. 2010;19:859-71.

Bajo R, Pusil S, Lopez ME, Canuet L, Pereda E, Osipova D, et al. Scopolamine effects on functional brain connectivity: a pharmacological model of Alzheimer's disease. Sci Rep. 2015;5:9748.

Besthorn C, Forstl H, Geiger-Kabisch C, Sattel H, Gasser T, Schreiter-Gasser U. EEG coherence in Alzheimer disease. Electroencephalogr Clin Neurophysiol. 1994;90:242-5.

Bian Z, Li Q, Wang L, Lu C, Yin S, Li X. Relative power and coherence of EEG series are related to amnestic mild cognitive impairment in diabetes. Front Aging Neurosci. 2014;6:11.

Bizzarro A, Marra C, Acciarri A, Valenza A, Tiziano FD, Brahe C, et al. Apolipoprotein E epsilon4 allele differentiates the clinical response to donepezil in Alzheimer's disease. Dement Geriatr Cogn Disord. 2005;20:254-61.

Blinowska KJ, Kaminski M. Functional brain networks: random, "small world" or deterministic? PLoS One. 2013;8:e78763.

Braekhus A, Ulstein I, Wyller TB, Engedal K. The Memory Clinic--outpatient assessment when dementia is suspected. Tidsskr Nor Laegeforen. 2011;131:2254-7.

Brunovsky M, Matousek M, Edman A, Cervena K, Krajca V. Objective assessment of the degree of dementia by means of EEG. Neuropsychobiology. 2003;48:19-26.

Chen CC, Hsu CY, Chiu HW, Hu CJ, Lee TC. Frequency power and coherence of electroencephalography are correlated with the severity of Alzheimer's disease: A multicenter analysis in Taiwan. J Formos Med Assoc. 2015;114:729-35.

Choi SH, Kim SY, Na HR, Kim BK, Yang DW, Kwon JC, et al. Effect of ApoE genotype on response to donepezil in patients with Alzheimer's disease. Dement Geriatr Cogn Disord. 2008;25:445-50.

Claus JJ, Strijers RL, Jonkman EJ, Ongerboer de Visser BW, Jonker C, Walstra GJ, et al. The diagnostic value of electroencephalography in mild senile Alzheimer's disease. Clin Neurophysiol. 1999;110:825-32.

Cook IA, Leuchter AF. Synaptic dysfunction in Alzheimer's disease: clinical assessment using quantitative EEG. Behav Brain Res. 1996;78:15-23.

Dauwels J, Vialatte F, Musha T, Cichocki A. A comparative study of synchrony measures for the early diagnosis of Alzheimer's disease based on EEG. Neuroimage. 2010;49:668-93.

Delorme A, Makeig S. EEGLAB: an open source toolbox for analysis of single-trial EEG dynamics including independent component analysis. J Neurosci Methods. 2004;134:9-21.

Dubois B, Feldman HH, Jacova C, Cummings JL, Dekosky ST, Barberger-Gateau P, et al. Revising the definition of Alzheimer's disease: a new lexicon. Lancet Neurol. 2010;9:1118-27.

Dunkin JJ, Leuchter AF, Newton TF, Cook IA. Reduced EEG coherence in dementia: state or trait marker? Biol Psychiatry. 1994;35:870-9.

Engedal K, Snaedal J, Hoegh P, Jelic V, Bo Andersen B, Naik M, et al. Quantitative EEG Applying the Statistical Recognition Pattern Method: A Useful Tool in Dementia Diagnostic Workup. Dement Geriatr Cogn Disord. 2015;40:1-12.

Folstein MF, Folstein SE, McHugh PR. "Mini-mental state". A practical method for grading the cognitive state of patients for the clinician. J Psychiatr Res. 1975;12:189-98.

Fraga FJ, Falk TH, Kanda PA, Anghinah R. Characterizing Alzheimer's disease severity via resting-awake EEG amplitude modulation analysis. PLoS One. 2013;8:e72240. 
Frisoni GB, Bocchetta M, Chetelat G, Rabinovici GD, de Leon MJ, Kaye J, et al. Imaging markers for Alzheimer disease: which vs how. Neurology. 2013;81:487-500.

Grosse P, Cassidy MJ, Brown P. EEG-EMG, MEG-EMG and EMG-EMG frequency analysis: physiological principles and clinical applications. Clin Neurophysiol. 2002;113:1523-31.

Hardmeier M, Hatz F, Bousleiman H, Schindler C, Stam CJ, Fuhr P. Reproducibility of functional connectivity and graph measures based on the phase lag index (PLI) and weighted phase lag index (WPLI) derived from high resolution EEG. PLoS One. 2014;9:e108648.

Jelic V, Johansson SE, Almkvist O, Shigeta M, Julin P, Nordberg A, et al. Quantitative electroencephalography in mild cognitive impairment: longitudinal changes and possible prediction of Alzheimer's disease. Neurobiol Aging. 2000;21:533-40.

Jelic V, Julin P, Shigeta M, Nordberg A, Lannfelt L, Winblad B, et al. Apolipoprotein E epsilon4 allele decreases functional connectivity in Alzheimer's disease as measured by EEG coherence. J Neurol Neurosurg Psychiatry. 1997;63:59-65.

Jelic V, Shigeta M, Julin P, Almkvist O, Winblad B, Wahlund LO. Quantitative electroencephalography power and coherence in Alzheimer's disease and mild cognitive impairment. Dementia. 1996;7:314-23.

Jelles B, Scheltens P, van der Flier WM, Jonkman EJ, da Silva FH, Stam CJ. Global dynamical analysis of the EEG in Alzheimer's disease: frequency-specific changes of functional interactions. Clin Neurophysiol. 2008;119:837-41.

Jeong J. EEG dynamics in patients with Alzheimer's disease. Clin Neurophysiol. 2004;115:1490-505. Knott V, Mohr E, Mahoney C, llivitsky V. Electroencephalographic coherence in Alzheimer's disease: comparisons with a control group and population norms. J Geriatr Psychiatry Neurol. 2000;13:1-8. Korf ES, Wahlund LO, Visser PJ, Scheltens P. Medial temporal lobe atrophy on MRI predicts dementia in patients with mild cognitive impairment. Neurology. 2004;63:94-100.

Lee TW, Girolami M, Sejnowski TJ. Independent component analysis using an extended infomax algorithm for mixed subgaussian and supergaussian sources. Neural Comput. 1999;11:417-41.

Lehmann C, Koenig T, Jelic V, Prichep L, John RE, Wahlund LO, et al. Application and comparison of classification algorithms for recognition of Alzheimer's disease in electrical brain activity (EEG). J Neurosci Methods. 2007;161:342-50.

Leuchter AF, Newton TF, Cook IA, Walter DO, Rosenberg-Thompson S, Lachenbruch PA. Changes in brain functional connectivity in Alzheimer-type and multi-infarct dementia. Brain. 1992;115:1543-61.

Leuchter AF, Spar JE, Walter DO, Weiner H. Electroencephalographic spectra and coherence in the diagnosis of Alzheimer's-type and multi-infarct dementia. A pilot study. Arch Gen Psychiatry. 1987;44:9938.

Locatelli T, Cursi M, Liberati D, Franceschi M, Comi G. EEG coherence in Alzheimer's disease.

Electroencephalogr Clin Neurophysiol. 1998;106:229-37.

McKhann G, Drachman D, Folstein M, Katzman R, Price D, Stadlan EM. Clinical diagnosis of Alzheimer's disease: report of the NINCDS-ADRDA Work Group under the auspices of Department of Health and Human Services Task Force on Alzheimer's Disease. Neurology. 1984;34:939-44.

Moretti DV, Frisoni GB, Pievani M, Rosini S, Geroldi C, Binetti G, et al. Cerebrovascular disease and hippocampal atrophy are differently linked to functional coupling of brain areas: an EEG coherence study in $\mathrm{MCl}$ subjects. J Alzheimers Dis. 2008;14:285-99.

Morris JC, Mohs RC, Rogers H, Fillenbaum G, Heyman A. Consortium to establish a registry for Alzheimer's disease (CERAD) clinical and neuropsychological assessment of Alzheimer's disease. Psychopharmacol Bull. 1988;24:641-52.

Mosconi L, Mistur R, Switalski R, Tsui WH, Glodzik L, Li Y, et al. FDG-PET changes in brain glucose metabolism from normal cognition to pathologically verified Alzheimer's disease. Eur J Nucl Med Mol Imaging. 2009;36:811-22.

Musaeus CS, Engedal K, Hgh P, Jelic V, Mrup M, Naik M, et al. EEG Theta Power Is an Early Marker of Cognitive Decline in Dementia due to Alzheimer's Disease. J Alzheimers Dis. 2018 a. 
Musaeus CS, Nielsen MS, Osterbye NN, Hogh P. Decreased Parietal Beta Power as a Sign of Disease Progression in Patients with Mild Cognitive Impairment. J Alzheimers Dis. 2018b;65:475-87.

Nolte G, Bai O, Wheaton L, Mari Z, Vorbach S, Hallett M. Identifying true brain interaction from EEG data using the imaginary part of coherency. Clin Neurophysiol. 2004;115:2292-307.

Ommundsen N, Engedal K, Oksengard AR. Validity of the quantitative EEG statistical pattern recognition method in diagnosing Alzheimer's disease. Dement Geriatr Cogn Disord. 2011;31:195-201.

Oostenveld R, Fries P, Maris E, Schoffelen JM. FieldTrip: Open source software for advanced analysis of MEG, EEG, and invasive electrophysiological data. Comput Intell Neurosci. 2011;2011:156869.

Patterson CE, Todd SA, Passmore AP. Effect of apolipoprotein E and butyrylcholinesterase genotypes on cognitive response to cholinesterase inhibitor treatment at different stages of Alzheimer's disease. Pharmacogenomics J. 2011;11:444-50.

Petersen RC, Thomas RG, Grundman M, Bennett D, Doody R, Ferris S, et al. Vitamin E and donepezil for the treatment of mild cognitive impairment. N Engl J Med. 2005;352:2379-88.

Reiman EM. Alzheimer's disease and other dementias: advances in 2013. Lancet Neurol. 2014;13:3-5. Resnick SM, Sojkova J, Zhou Y, An Y, Ye W, Holt DP, et al. Longitudinal cognitive decline is associated with fibrillar amyloid-beta measured by [11C]PiB. Neurology. 2010;74:807-15.

Sankari Z, Adeli H, Adeli A. Intrahemispheric, interhemispheric, and distal EEG coherence in Alzheimer's disease. Clin Neurophysiol. 2011;122:897-906.

Schmidt M. minFunc: unconstrained differentiable multivariate optimization in Matlab.

2005:http://www.cs.ubc.ca/ schmidtm/Software/minFunc.html.

Shulman Kl. Clock-drawing: is it the ideal cognitive screening test? Int J Geriatr Psychiatry. 2000;15:548-61.

Sloan EP, Fenton GW, Standage KP. Anticholinergic drug effects on quantitative electroencephalogram, visual evoked potential, and verbal memory. Biol Psychiatry. 1992;31:600-6.

Snaedal J, Johannesson GH, Gudmundsson TE, Blin NP, Emilsdottir AL, Einarsson B, et al. Diagnostic accuracy of statistical pattern recognition of electroencephalogram registration in evaluation of cognitive impairment and dementia. Dement Geriatr Cogn Disord. 2012;34:51-60.

Trambaiolli LR, Spolaor N, Lorena AC, Anghinah R, Sato JR. Feature selection before EEG classification supports the diagnosis of Alzheimer's disease. Clin Neurophysiol. 2017;128:2058-67.

Vinck M, Oostenveld R, van Wingerden M, Battaglia F, Pennartz CM. An improved index of phasesynchronization for electrophysiological data in the presence of volume-conduction, noise and sample-size bias. Neuroimage. 2011;55:1548-65.

Visser PJ, Verhey F, Knol DL, Scheltens P, Wahlund LO, Freund-Levi Y, et al. Prevalence and prognostic value of CSF markers of Alzheimer's disease pathology in patients with subjective cognitive impairment or mild cognitive impairment in the DESCRIPA study: a prospective cohort study. Lancet Neurol. 2009;8:619-27. Wada Y, Nanbu Y, Kikuchi M, Koshino Y, Hashimoto T, Yamaguchi N. Abnormal functional connectivity in Alzheimer's disease: intrahemispheric EEG coherence during rest and photic stimulation. Eur Arch Psychiatry Clin Neurosci. 1998;248:203-8.

Wang R, Wang J, Yu H, Wei X, Yang C, Deng B. Decreased coherence and functional connectivity of electroencephalograph in Alzheimer's disease. Chaos. 2014;24:033136.

Winblad B, Palmer K, Kivipelto M, Jelic V, Fratiglioni L, Wahlund LO, et al. Mild cognitive impairment-beyond controversies, towards a consensus: report of the International Working Group on Mild Cognitive Impairment. J Intern Med. 2004;256:240-6.

Xia M, Wang J, He Y. BrainNet Viewer: a network visualization tool for human brain connectomics. PLoS One. 2013;8:e68910. 
Table 1. Characteristics of study participants.

\begin{tabular}{|c|c|c|c|c|}
\hline & $\begin{array}{c}\mathbf{H C} \\
(n=135)\end{array}$ & $\begin{array}{c}\mathbf{A D} \\
(n=117)\end{array}$ & $\begin{array}{c}\text { MCI } \\
(n=117)\end{array}$ & $\begin{array}{c}p \text {-value } \\
\text { ADvs.MCIvs. HC }\end{array}$ \\
\hline Mean age $(S D)$, years & $66.44(7.64)$ & $75.49(7.65)$ & $70.15(8.13)$ & $<0.000$ \\
\hline Sex (female), \% & 60.7 & 60.7 & 53.0 & 0.377 \\
\hline Education, years $(S D)$ & $13.89(3.61)$ & $10.07(3.39)$ & $11.57(3.92)$ & $<0.000$ \\
\hline Antipsychotics & 0 & 4 & 1 & \\
\hline Antidepressants & 0 & 18 & 25 & \\
\hline Tranquilizers/hypnotics & 3 & 6 & 12 & \\
\hline Anti-dementia drugs & 0 & 18 & 2 & \\
\hline Painkillers & 4 & 4 & 6 & \\
\hline Lumbal punctures performed & & 32 & 38 & \\
\hline Amlyoid beta 42, mean $(S D)$ & & $516.34 \quad(114.52)$ & $876.16 \quad(354.92)$ & $<0.000$ \\
\hline Total, tau mean $(S D)$ & & $532.31 \quad(273.85)$ & $420.98 \quad(230.51)$ & 0.069 \\
\hline Phosphorylated tau, mean $(S D)$ & & $106.33(114.52)$ & $63.16(26.72)$ & 0.027 \\
\hline$M M S E$, mean $(S D)$ & $28.91(1.34)$ & $23.52(3.79)$ & $27.11(2.16)$ & $<0.000$ \\
\hline Word List Memory, mean $(S D)$ & $20.49(3.99)$ & $11.06(3.83)$ & $15.25(4.29)$ & $<0.000$ \\
\hline Word List Recognition, mean $(S D)$ & $7.39(1.69)$ & $1.52(1.64)$ & $3.66(2.34)$ & $<0.000$ \\
\hline Word List Recall, mean $(S D)$ & $19.47(1.44)$ & $15.84(2.62)$ & $17.92(2.01)$ & $<0.000$ \\
\hline CERAD Word List, mean (SD) & $47.46(5.55)$ & $28.47(6.44)$ & $36.84(7.39)$ & $<0.000$ \\
\hline
\end{tabular}

$\mathrm{HC}=$ healthy controls, $\mathrm{AD}=$ Alzheimer's disease, $\mathrm{MCI}=$ mild cognitive impairment, $\mathrm{SD}=$ standard deviation, $\mathrm{MMSE}=\mathrm{Mini}-\mathrm{Mental}$

State Examination. P-values show the differences when comparing patients with Alzheimer's disease, mild cognitive controls, and healthy controls. A p-value was considered significant when $\mathrm{p}$-value $<0.05$. 
Fig 1. Flow diagram of the number of included participants in the current study and the excluded participants after preprocessing. 
Fig 2. Confusion matrices displaying results from the prediction analyses for A) coherence with eyes closed, B) imaginary part of coherence with eyes closed, C) weighted phase-lag index with eyes closed, D) coherence with eyes open, E) imaginary part of coherence with eyes open, F) weighted phase-lag index with eyes closed and G) coherence in the alpha band for eyes closed. $\mathrm{HC}=$ healthy controls, $\mathrm{AD}=$ Alzheimer's disease, $\mathrm{MCI}=$ mild cognitive impairment. 
Fig 3. Difference in coherence between groups (Alzheimer's disease vs. healthy controls; mild cognitive impairment vs. healthy controls; and Alzheimer's disease vs. mild cognitive impairment) for all seven frequency bands (A-G) with eyes closed. The difference is illustrated if the post-hoc test was significant $(<0.001)$, and t-values were used to illustrate the differences. Red indicates increased and blue decreased coherence in the first group listed (i.e., decreased alpha coherence between Alzheimer's disease and healthy controls can be found in row $\mathrm{C}$ column one, $\mathrm{AD}$ vs. $\mathrm{HC}$ ). $\mathrm{HC}=$ healthy controls, $\mathrm{AD}=$ Alzheimer's disease, $\mathrm{MCI}=$ mild cognitive impairment. 
Fig 4. Total coherence in each frequency band for eyes closed. The comparison is considered significant if the ANCOVA was significant and the post-hoc t-test was significant (p-value $<0.05) . * 1=$ significant differences between all groups; *2 = significant difference between $\mathrm{MCI}$ and both $\mathrm{HC}$ and $\mathrm{AD} . \mathrm{HC}=$ healthy controls, $\mathrm{AD}=$ Alzheimer's disease, $\mathrm{MCI}$ $=$ mild cognitive impairment. 
Fig 5. Difference in coherence between groups (Alzheimer's disease vs. healthy controls; mild cognitive impairment vs. healthy controls; and Alzheimer's disease vs. mild cognitive impairment) for the delta, theta, alpha, and beta bands (AD) with eyes open. The difference is illustrated if the post-hoc test was significant $(<0.001)$, and t-values were used to illustrate the differences. Red indicates increased and blue decreased coherence in the first group listed. $\mathrm{HC}=$ healthy controls, $\mathrm{AD}=$ Alzheimer's disease, $\mathrm{MCI}=$ mild cognitive impairment 
Fig 6. Total coherence in each frequency band for eyes open. The comparison is considered significant if the ANCOVA was significant and the post-hoc t-test was significant ( $\mathrm{p}$-value $<0.05) . * 1=$ significant differences between all groups; *2 = significant difference between MCI and both $\mathrm{HC}$ and $\mathrm{AD} ; * 3=$ significant difference between $\mathrm{HC}$ and both MCI and $\mathrm{AD} ; * 4=$ significant difference between MCI and $\mathrm{HC} . \mathrm{HC}=$ healthy controls; $\mathrm{AD}=$ Alzheimer's disease; $\mathrm{MCI}=$ mild cognitive impairment. 


\begin{tabular}{|c|c|c|c|c|}
\hline & $\mathrm{HC}$ & MCI & AD & $p$-value \\
\hline & $(n=135)$ & $(n=117)$ & $(n=117)$ & $\mathrm{AD}$ vs $\mathrm{MCI}$ vs $\mathrm{HC}$ \\
\hline Mean age (SD), years & $\begin{array}{l}66.44 \\
(7.64)\end{array}$ & $70.15(8.13)$ & $75.49(7.65)$ & $<0.000$ \\
\hline Sex (female), $\%$ & 60.7 & 53 & 60.7 & 0.377 \\
\hline Education, years (SD) & $\begin{array}{l}13.89 \\
(3.61) \\
\end{array}$ & $11.57(3.92)$ & $10.07(3.39)$ & $<0.000$ \\
\hline Antipsychotics & 0 & 1 & 4 & 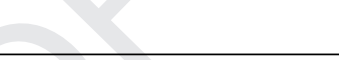 \\
\hline Antidepressants & 0 & 25 & 18 & \\
\hline Tranquilizers/hypnotics & 3 & 12 & 6 & 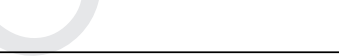 \\
\hline Anti-dementia drugs & 0 & 2 & 18 & \\
\hline Painkillers & 4 & 6 & 8 & \\
\hline Lumbar punctures performed & & 38 & 32 & \\
\hline Amyloid beta 42, mean (SD) & & $\begin{array}{l}876.16 \\
(354.92)\end{array}$ & \begin{tabular}{|l|}
516.34 \\
$(114.52)$
\end{tabular} & $<0.000$ \\
\hline Total tau, mean (SD) & & $\begin{array}{l}420.98 \\
(230.51)\end{array}$ & $\begin{array}{l}532.31 \\
(273.85)\end{array}$ & 0.069 \\
\hline Phosphorylated tau, mean (SD) & & $63.16(26.72)$ & $\begin{array}{l}106.33 \\
(114.52) \\
\end{array}$ & 0.027 \\
\hline MMSE, mean (SD) & $\begin{array}{l}28.91 \\
(1.34)\end{array}$ & $27.11(2.16)$ & $23.52(3.79)$ & $<0.000$ \\
\hline Word list memory, mean (SD) & $\begin{array}{l}20.49 \\
(3.99)\end{array}$ & $15.25(4.29)$ & $11.06(3.83)$ & $<0.000$ \\
\hline Word list recall, mean (SD) & $7.39(1.69)$ & $3.66(2.34)$ & $1.52(1.64)$ & $<0.000$ \\
\hline $\begin{array}{l}\text { Word list recognition, mean } \\
\text { (SD) }\end{array}$ & $\begin{array}{l}19.47 \\
(1.44)\end{array}$ & $17.92(2.01)$ & $15.84(2.62)$ & $<0.000$ \\
\hline CERAD Word List, mean (SD) & $\begin{array}{l}47.46 \\
(5.55)\end{array}$ & $36.84(7.39)$ & $28.47(6.44)$ & $<0.000$ \\
\hline
\end{tabular}




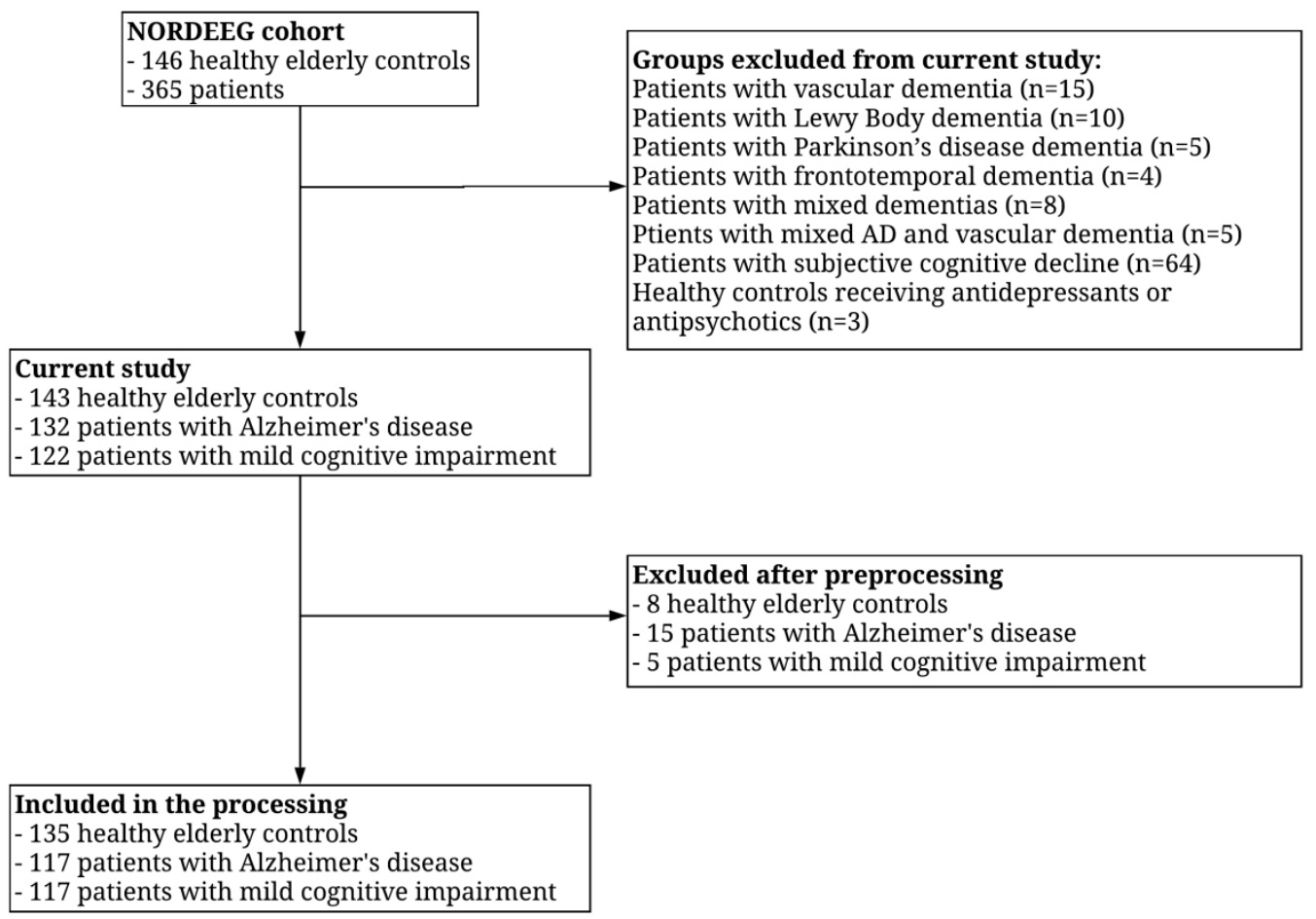



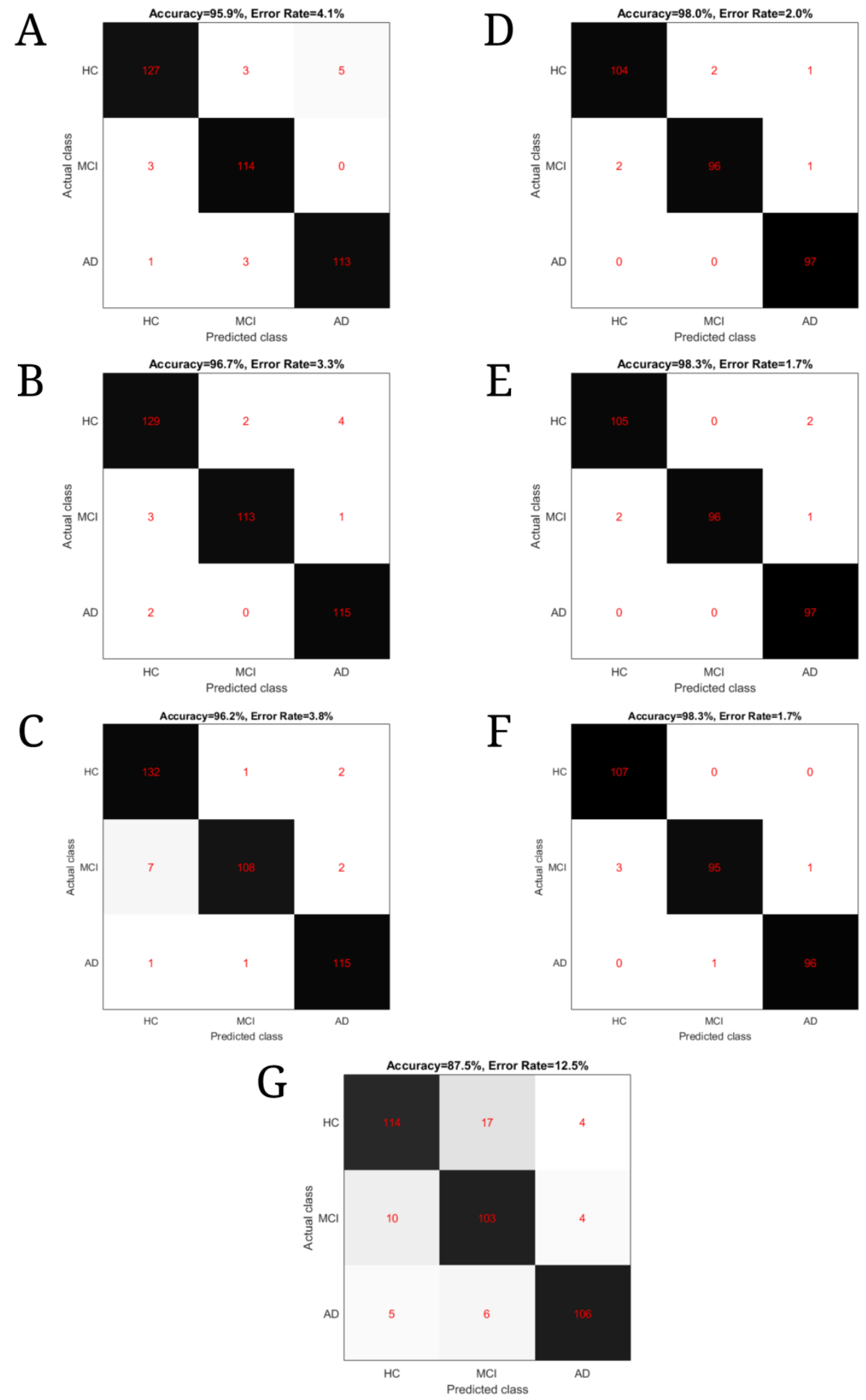
A

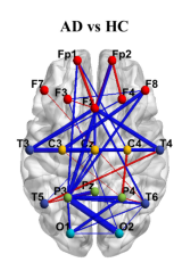

Delta (1-3.99 Hz)
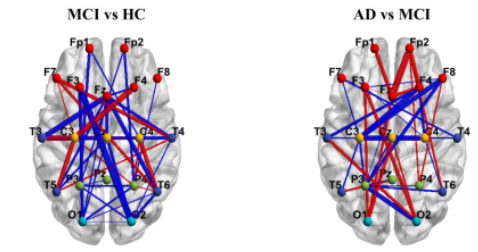

B

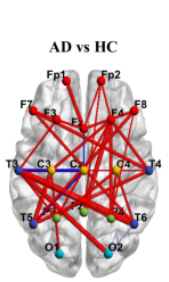

Theta (4-7.99 Hz)
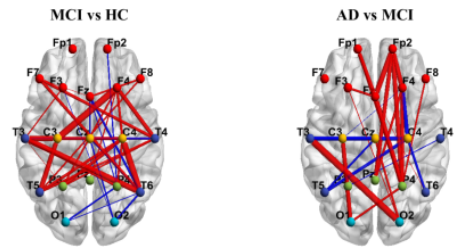

C

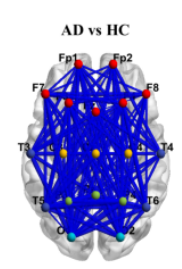

Alpha (8-12.99 Hz)
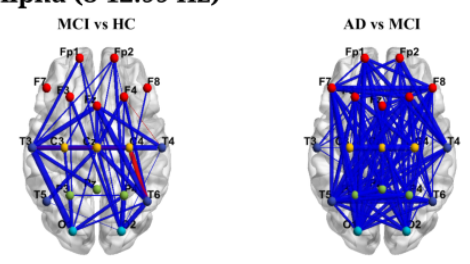

D

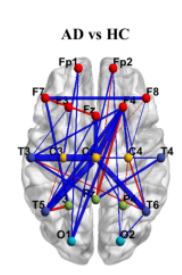

Beta1 (13-17.99 Hz)
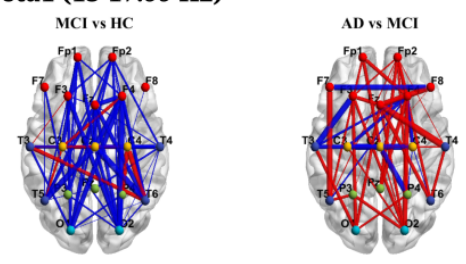

E

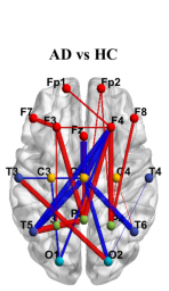

Beta2 (18-23.99 Hz)
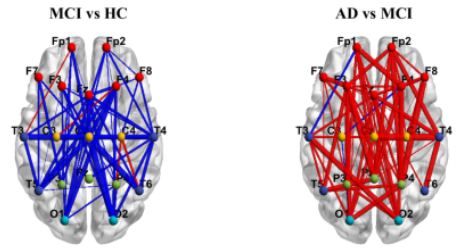

$\mathbf{F}$

Beta3 (24-29.99 Hz)
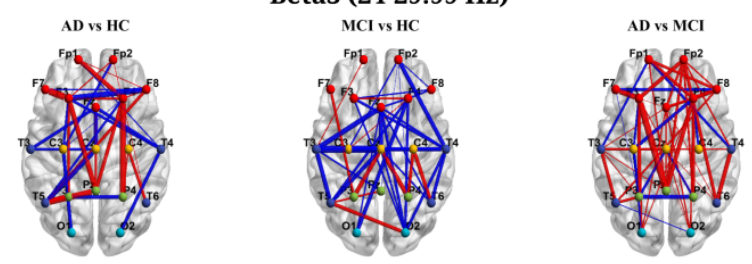

G

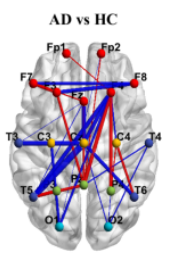

Beta (13-29.99 Hz)
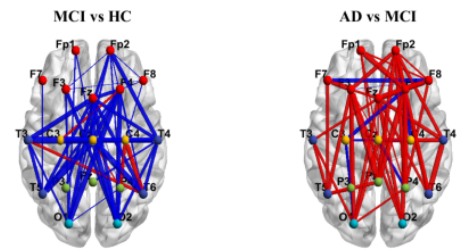


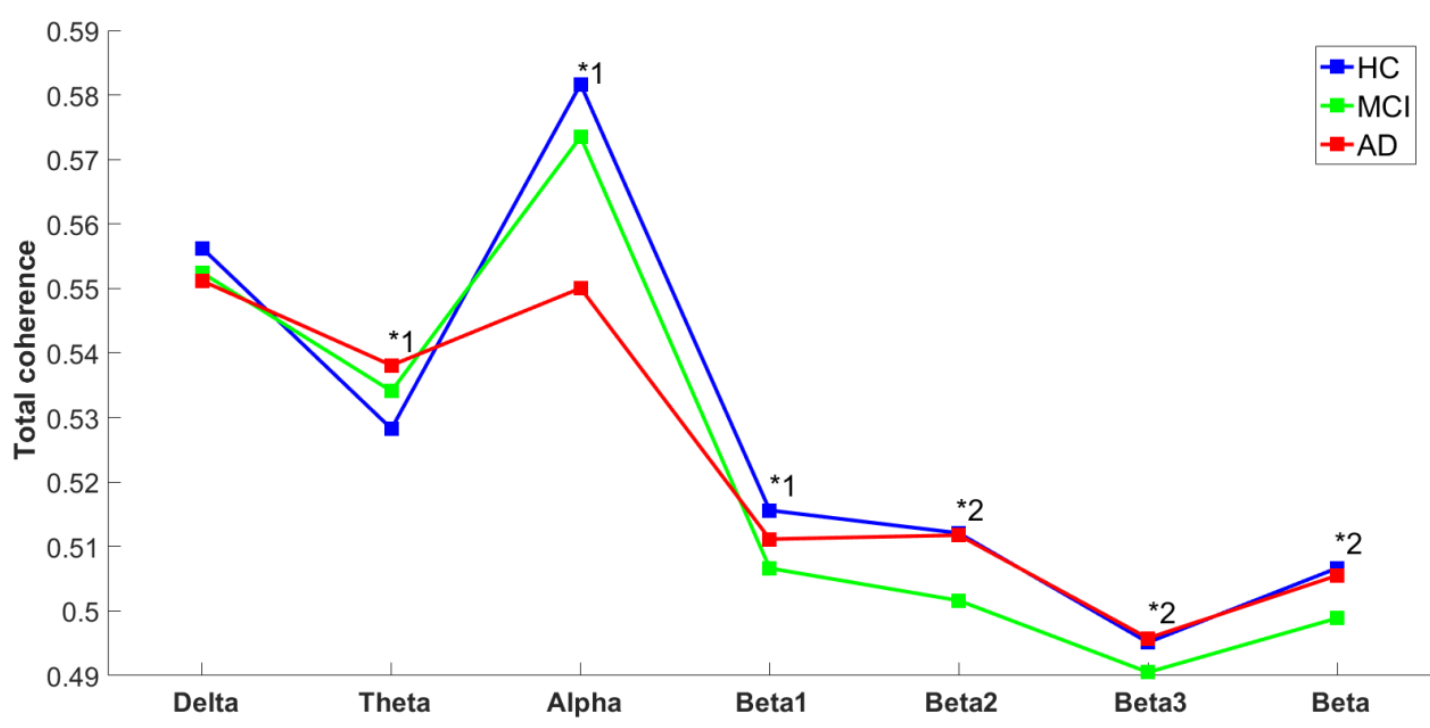


A

Delta (1-3.99 Hz)
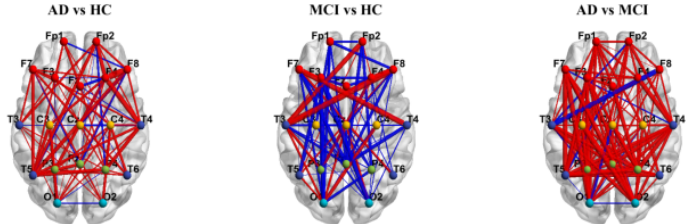

B

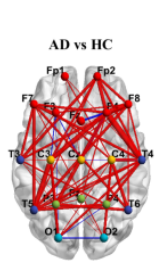

Theta (4-7.99 Hz)
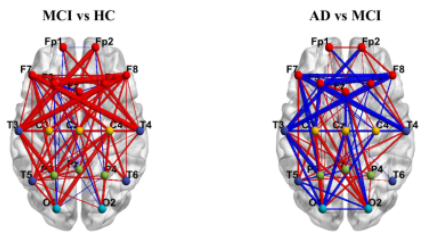

C

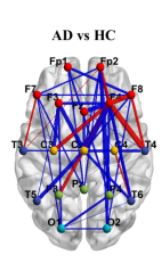

Alpha (8-12.99 Hz)
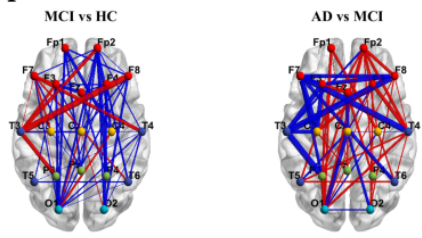

D

Beta1 (13-17.99 Hz)
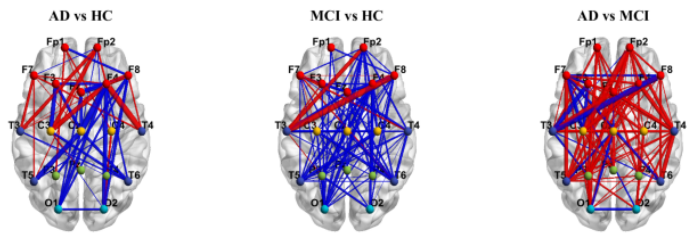

E

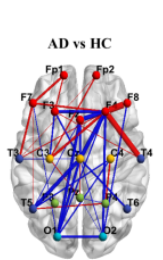

Beta2 (18-23.99 Hz)
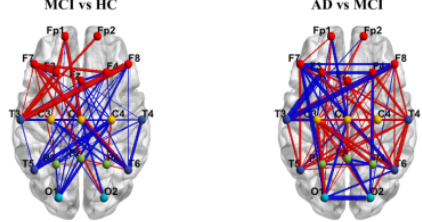

$\mathbf{F}$

Beta3 (24-29.99 Hz)
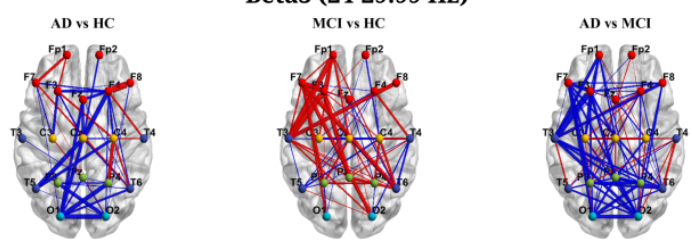

G

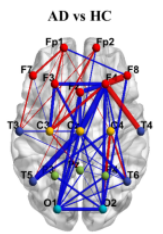

Beta (13-29.99 Hz)
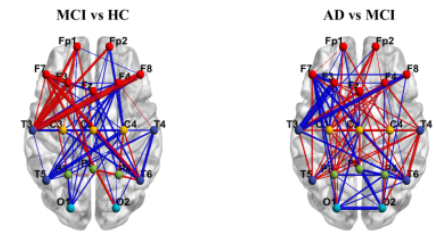


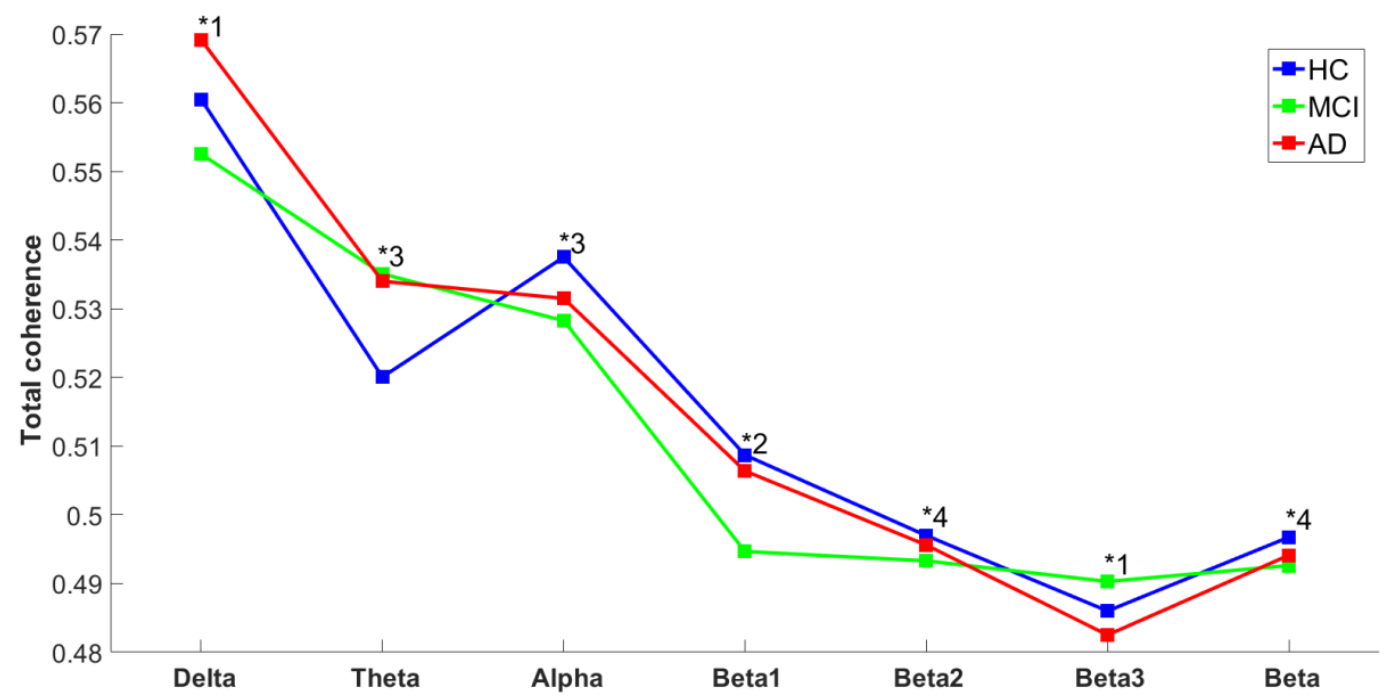

